\title{
Hole spin mode locking and coherent dynamics in a largely inhomogeneous ensemble of $p$-doped InAs quantum dots
}

\author{
F. Fras,,${ }^{1, *}$ B. Eble, ${ }^{1}$ B. Siarry, ${ }^{1}$ F. Bernardot,${ }^{1}$ A. Miard, ${ }^{2}$ A. Lemaître, ${ }^{2}$ C. Testelin,,${ }^{1}$ and M. Chamarro ${ }^{1}$ \\ ${ }^{1}$ Institut des NanoSciences de Paris, UPMC Université Paris 06, CNRS UMR 7588, 4 Place Jussieu, F-75252 Paris Cedex 05, France \\ ${ }^{2}$ Laboratoire de Photonique et Nanostructures, CNRS, Route de Nozay, F-91460 Marcoussis, France
}

(Received 30 July 2012; published 19 October 2012)

\begin{abstract}
In this study, we evidence hole spin mode locking in a largely inhomogeneous $p$-doped InAs quantum dot (QD) ensemble, $\Delta g_{x}^{h} / g_{x}^{h} \approx 34 \%$, which allows us to reveal a long spin coherence time of $T_{2}^{h} \approx 0.8 \mu \mathrm{s}$. In addition, with a two-pump experiment, we demonstrate, in a low magnetic range 60-120 $\mathrm{mT}$, the possibility to synchronize and tune a single subset of QDs through the distribution. Experiments are supported by an analysis within the density matrix approach.
\end{abstract}

PACS number(s): 78.67.Hc, 78.47.jh

The resident spin of a carrier confined in a quantum dot (QD) is a promising candidate for quantum information systems. Until recently, most theoretical and experimental studies have been centered on the electron spin, and have demonstrated that the ultimate limitation, at low temperature and zero magnetic field, is the hyperfine interaction of an electron with a nuclear-spin ensemble. Due to the limited number of QD nuclei interacting with the electron spin, on the order of $10^{5}$, random fluctuations of the nuclearspin bath lead to a dephasing time on the order of ns for InAs QDs. Recently, the initialization of hole spin has been demonstrated, ${ }^{1-7}$ and it has emerged as a more attractive candidate, with a one-order-of-magnitude weaker hyperfine coupling. ${ }^{4,8-12}$ Several studies have reported a long hole spin relaxation time $T_{1}^{h}$, from $\mu$ s to $\mathrm{ms}$, under different conditions of temperature and magnetic field. ${ }^{1,13-16}$ In early single-hole experiments of coherent population trapping, the hole spin coherence time was estimated to be larger than 100 ns. ${ }^{17}$ In more recent experiments in time domain, the hole-spin coherence time has been found to be $\leqslant 20 \mathrm{~ns}$, but these single hole-spin measurements were affected by electrical-noise ${ }^{18,19}$ or nuclear-spin ${ }^{20}$ fluctuations. Recent spin-echo experiments allow to circumvent the inhomogeneous decoherence and measure a much longer hole-spin coherence time $T_{2}^{h}$, in the microsecond range between 6 and $10 \mathrm{~T}^{18}$ This value is of the same order of magnitude as for electrons ${ }^{21}$ and represents an upper bound on the free-induction decay time of hole spin coherence. $^{22}$

For electron spins, the possibility to go beyond the ensemble inhomogeneities and to measure a long coherence time by using a periodic train of circularly polarized pulses has been demonstrated. A single pulse creates an ensemble of electron spins along its propagation direction and perpendicular to an external magnetic field (Voigt geometry). Each electron spin precesses about the field and, due to the inhomogeneity of the precession frequencies, the phase coherence is quickly lost. The train of pulses allows the phase synchronization of subsets of the whole spin ensemble, for which the precession frequency is a multiple of the laser repetition rate. This effect was called "electron spin mode locking."23,24 It has allowed the measurement of an electron spin coherence time of $600 \mathrm{~ns}$ at $2 \mathrm{~T}^{25}$ and $3 \mu \mathrm{s}$ at $6 \mathrm{~T},{ }^{23}$ in an ensemble of InGaAs QDs with a relative dispersion of the in-plane electron Landé factor $\Delta g_{x}^{e} / g_{x}^{e} \approx 1 \%$.
Holes are more sensitive than electrons to the anisotropy of local electrostatic fields, strains, and geometrical asymmetry of the confinement potentials, especially the in-plane Landé factor $g_{x, y}^{h}{ }^{18-20,26,27}$ This leads to a broad distribution of $g_{x, y}^{h}$ and may prevent the observation of a hole spin synchronization signal. In this Rapid Communication, we evidence the mode locking of hole spins in a largely inhomogeneous ensemble of $p$-doped InAs/GaAs QDs with a relative dispersion $\Delta g_{x}^{h} / g_{x}^{h} \approx$ $34 \%$. As expected, when the hole spin dephasing time is controlled by the $g_{x}^{h}$ inhomogeneities, we found a linear dependence of the hole-spin dephasing rate $1 / T_{h}^{*}$ on the magnetic field, and we then extracted information on $\Delta g_{x}^{h}$. We show that, as for the electron spin, ${ }^{23,28}$ the coherent response of the synchonized QD assembly can be controlled and amplified by a second laser pulse. Finally, a model of coupled trion and hole spin dynamics enables to estimate a lower bound to $T_{2}^{h}=0.8 \mu \mathrm{s}$, which is in good agreement with recent spin-echo measurements ${ }^{18}$ and is much longer than the time obtained in single-hole experiments. ${ }^{19,20}$

The $p$-doped QD structures were grown by molecular beam epitaxy and have been described elsewhere. ${ }^{9}$ The sample was placed at $2 \mathrm{~K}$ in a cryostat containing a split superconducting coil which allows the application of a magnetic field in the plane of QDs. A train of 2-ps pulses from a 76-MHz mode-locked Ti:sapphire laser, tuned to the trion transition at $1.36 \mathrm{eV}$, is split into pump and probe beams propagating along the growth direction (i.e., $z$ ). One or two pump beams excite the sample, their polarization being $\sigma^{+} / \sigma^{-}$modulated at $500 \mathrm{kHz}$ with an electro-optic modulator. The pump spot radius $(\approx 30 \mu \mathrm{m})$ on the sample is slightly larger than the spot of the probe beam. The probe beam is linearly polarized and modulated at $370 \mathrm{~Hz}$; after probe transmission through the sample, the photoinduced circular dichroism (PCD) signal is measured (see Ref. 2).

Figures 1(a) and 1(b) show the PCD signal obtained at $2 \mathrm{~K}$ in $p$-doped InAs/GaAs QDs under magnetic field $B_{x}$ : The PCD signal oscillates and is rapidly damped. At positive pump-probe delays, the PCD signal associates contributions from the photocreated electron spins in the trion states and from the resident-hole spins. At negative delays, the nonzero signal $\operatorname{PCD}(t<0)$ is a signature of the coherent evolution of the resident-hole spin. The oscillation amplitude at $t<0$ is less pronounced but clearly observed [see Fig. 1(b)]. $\operatorname{PCD}(t<0)$ is due to the QD subsets which interfere in a constructive 


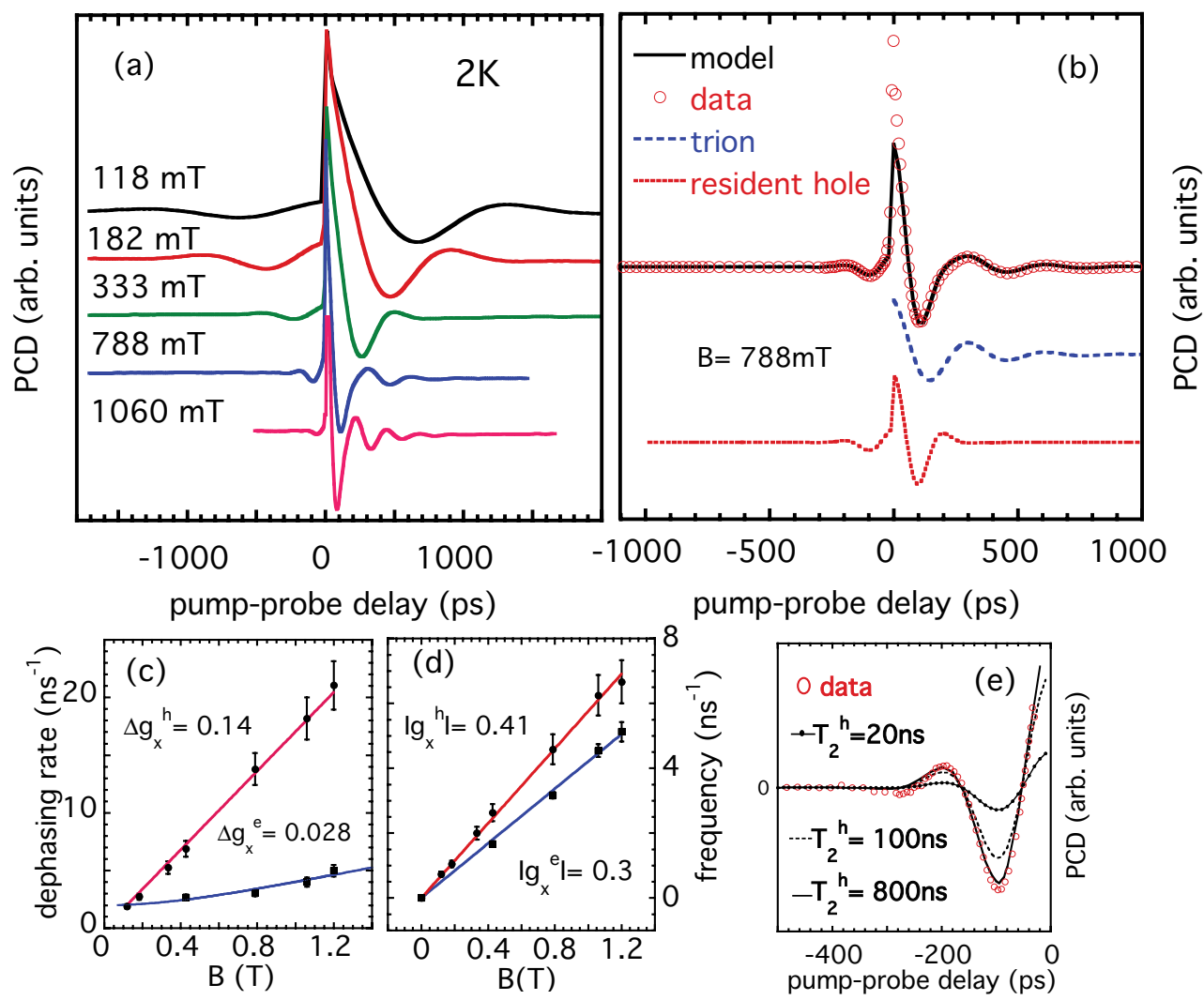

FIG. 1. (Color online) (a) PCD signal at $2 \mathrm{~K}$ vs pump-probe delay for different $B_{x}$ values and an excitation density of $2 \mathrm{~mW}$. (b) PCD signal at $788 \mathrm{mT}$ : Data (open symbols) and theoretical curve (solid line; parameters: $g_{x}^{e}=-0.31, g_{x}^{h}=0.41, T_{\Delta}^{e}=500 \mathrm{ps}, \Delta g_{x}^{e} / g_{x}^{e}=9 \%$, $\Delta g_{x}^{h} / g_{x}^{h}=34 \%, T_{2}^{h}=800 \mathrm{~ns}$, and $\left.\theta=0.9 \pi\right)$. The trion-spin and resident-hole spin contributions are shown by the dashed and dotted lines, respectively. (c) and (d) Field dependence of $1 / T_{h}^{*}$ and $\omega_{h}$, and $1 / T_{e}^{*}$ and $\omega_{e}$ (circle and square symbols, respectively). Solid lines are fits (see text). (e) Magnification of the $\operatorname{PCD}(t<0)$ at $788 \mathrm{mT}$ : experimental data (open circles), and theoretical curves (lines) for different $T_{2}^{h}$.

way beyond $T_{h}^{*}$ because of their quasihomogeneous $T_{2}^{h}>T_{L}$ ( $T_{L}=13.1 \mathrm{~ns}$, the laser period). They fulfill the condition of phase synchronization for a hole spin, i.e., $\omega_{h}=\mu_{B} g_{x}^{h} B_{x} / \hbar=$ $2 \pi N / T_{L}$, where $N$ is an integer.

We can consider two main sources for the inhomogeneity of electron- and hole-spin ensembles. First, a confined carrier, an electron or hole, is subject to a hyperfine field due to nuclear-spin fluctuations which are different from QD to QD. ${ }^{10,29}$ The hyperfine dephasing time $T_{\Delta}^{e, h}$ is independent of $B_{x}$ and is on the order of 1 or $10 \mathrm{~ns}$ for the electron and hole, respectively. ${ }^{4,11,12}$ Second, the inhomogeneity of Landé factors leads to a spreading of the spin precession frequencies. Assuming a Gaussian distribution for both dephasing sources, the dephasing rate for electron and hole can be written as $1 / T_{e, h}^{*}\left(B_{x}\right)=\sqrt{\left[\left(1 / T_{\Delta}^{e, h}\right)^{2}+2\left(\mu_{B} \Delta g_{x}^{e, h} B_{x} / \hbar\right)^{2}\right]} .^{24}$

In order to obtain the mean-value parameters defining the PCD signal and dephasing, we have fitted $\operatorname{PCD}(t<0)$ to a damped cosine, $\operatorname{PCD}(t<0)=a+$ $b \cos \left(\omega_{h} t\right) \exp \left[-\left(t / 2 T_{h}^{*}\right)^{2}\right]$. The damping of the PCD oscillations at positive delays is due to two different contributions: the radiative recombination of trions $\left[\tau_{R}=800 \mathrm{ps}\right.$ (Ref. 9)] and the hole- and electron-spin ensemble inhomogeneities. We have fitted the $\operatorname{PCD}(t>0)$ curves beyond $T_{h}^{*}$ with the following expression: $\operatorname{PCD}\left(t>T_{h}^{*}\right)=a+$ $c \cos \left(\omega_{e} t\right) \exp \left[-\left(t / 2 T_{e}^{*}\right)^{2}\right] \exp \left[-\left(t / \tau_{R}\right)\right]$. Figure 1(c) shows the magnetic-field dependence of $1 / T_{e}^{*}$ and $1 / T_{h}^{*}$. For holes, the magnetic field meets the condition $B_{x} \gg \hbar / \mu_{B} \Delta g_{x}^{h} T_{\Delta}^{h}$ and $1 / T_{h}^{*}$ shows a linear $B_{x}$ dependence, $1 / T_{h}^{*}\left(B_{x}\right)=$ $\sqrt{2}\left(\mu_{B} \Delta g_{x}^{h} B_{x} / \hbar\right)$, with $\Delta g_{x}^{h}=0.14$. For electrons, $1 / T_{e}^{*}$ is determined by $1 / T_{\Delta}^{e}$ and $\Delta g_{x}^{e}$. The solid line in Fig. 1(c) represents a fit to the expression $1 / T_{e}^{*}$ with $T_{\Delta}^{e}=500 \mathrm{ps}{ }^{9}{ }^{9}$ we obtain $\Delta g_{x}^{e}=0.028$.

Figure 1(d) shows the linear dependence of $\omega_{e, h}$ on magnetic field; from the fitting solid lines, we obtain the values $\left|g_{x}^{e}\right|=0.3$ and $\left|g_{x}^{h}\right|=0.41$. We underline that a large number of QD subsets contribute to the $\operatorname{PCD}(t<0)$, and then we obtain a mean value of $\left|g_{x}^{h}\right|$. The measured $\left|g_{x}^{e}\right|$ and its dispersion $\Delta g_{x}^{e} /\left|g_{x}^{e}\right|$ are in good agreement with previous results reported on InAs QDs: ${ }^{27,30}$ meanwhile the $g_{x}^{h}$ value is relatively large and dispersed. That could be explained by the $g_{x}^{h}$ sensitivity to the structural parameters of QDs as discussed in Ref. 26. Indeed, recent $\mu$-luminescence experiments in similar InAs QDs show a large dispersion. ${ }^{31}$ Moreover, spinnoise experiments in ensembles of QDs (Ref. 32) lead to a value ranging from 0.05 to 0.2 for InGaAs QDs, emitting between 1.38 and $1.40 \mathrm{eV}$.

In parallel to this straightforward analysis of our PCD data, we have used the density matrix formalism to model the interconnected hole-trion spin dynamics, and we have treated, in a sequential way, the pump pulse interaction with 
the p-doped QDs, as well as the coherent free evolution between two consecutive pump pulses. A QD is described by four quantum states: The two hole ground states $\left|J_{z}= \pm 3 / 2\right\rangle$ are denoted by $\left|S_{z}^{h}=+1 / 2\right\rangle=|1\rangle$ and $\left|S_{z}^{h}=-1 / 2\right\rangle=|2\rangle$ in the pseudospin $1 / 2$ formalism, and the first excited (trion) states are denoted by $\left|S_{z}^{e}=-1 / 2\right\rangle=|3\rangle$ and $\left|S_{z}^{e}=1 / 2\right\rangle=$ $|4\rangle$. In the range $B_{x}=0-1.2 \mathrm{~T}$, the spectral width of the pulses is greater than the Zeeman splitting along the $x$ direction. A $\sigma^{+}$ps-pump pulse modifies the density matrix operator $\rho$ of the involved four-level system according to the unitary transformation $\rho\left(0^{+}\right)=U_{\sigma^{+}}(\theta) \rho\left(0^{-}\right) U_{\sigma^{+}}^{+}(\theta)$, where $U_{\sigma^{+}}(\theta)=\left(\begin{array}{cc}\cos \frac{\theta}{2} & -i \sin \frac{\theta}{2} \\ -i \sin \frac{\theta}{2} & \cos \frac{\theta}{2}\end{array}\right)_{|3\rangle,|2\rangle} \otimes \hat{1}_{|4\rangle,|1\rangle}$ and $\theta$ is the Rabi angle. The evolution between two pulses is described by using the von Neumann equation $\dot{\rho}=\frac{-i}{\hbar}\left[H_{Z}, \rho\right]+\ell[\rho]$, where $H_{Z}=$ $\frac{1}{2} \hbar \omega_{e} \sigma_{x}^{X+}+\frac{1}{2} \hbar \omega_{h} \sigma_{x}^{h}$ is the Zeeman Hamiltonian $\left(\hbar \omega_{j}=\right.$ $\left.g_{x}^{j} \mu_{B} B_{x}, j=e, h\right)$, in which $\sigma_{x}^{X+}$ (respectively $\sigma_{x}^{h}$ ) is the $x$ Pauli matrix associated with the trion spin (respectively the hole spin). The dissipator $\ell[\rho]$ is the sum of six contributions: Two terms are related to the spontaneous emission with the form $\gamma_{i}\left[2 c_{i} \rho c_{i}^{+}-\left\{c_{i}^{+} c_{i}, \rho\right\}_{+}\right]\left(\gamma_{1}=\gamma_{2}=\frac{1}{2 \tau_{R}}, c_{1}=|1\rangle\langle 4|\right.$, $\left.c_{2}=|2\rangle\langle 3|\right)$; four terms take into account the effective decoherence mechanisms acting on both trion and hole spins, with the form $\gamma_{i}\left[2 \bar{c}_{i} \rho \bar{c}_{i}^{+}-\left\{\bar{c}_{i}^{+} \bar{c}_{i}, \rho\right\}_{+}\right]\left(\gamma_{3}=\gamma_{4}=1 / 2 T_{e}^{*}, \bar{c}_{3}=\right.$ $|\overline{4}\rangle\langle\overline{3}|, \bar{c}_{4}=\bar{c}_{3}^{+}$, and $\left.\gamma_{5}=\gamma_{6}=1 / 2 T_{2}^{h}, \bar{c}_{5}=|\overline{2}\rangle\langle\overline{1}|, \bar{c}_{6}=\bar{c}_{5}^{+}\right)$ in the eigenstate basis, along the $x$ axis, $\left(\left|S_{x}^{h}=+1 / 2\right\rangle=\right.$ $|\overline{1}\rangle,\left|S_{x}^{h}=-1 / 2\right\rangle=|\overline{2}\rangle,\left|S_{x}^{e}=-1 / 2\right\rangle=|\overline{3}\rangle,\left|S_{x}^{e}=+1 / 2\right\rangle=$ $|\overline{4}\rangle)$. The trion Larmor oscillation damping of the photocreated electron spin ensemble is affected by $\Delta g_{x}^{e}$ and $1 / T_{\Delta}^{e}$, and is given by the expression $T_{e}^{*}\left(B_{x}\right)$, as already mentioned. The periodic optical excitation gives rise to a stationary regime which fulfills the condition $\rho\left(0^{-}\right)=\rho\left(T_{L}\right)$, whatever is the employed protocol (the one-pulse or the two-pulse one). Solving this equation gives the initial hole spin state, which is then injected in the kinetic equations. Finally, the PCD dynamics is deduced by computing PCD $\propto \int \operatorname{Tr}\left[\rho(t)\left(\sigma_{z}^{h}-\sigma_{z}^{X+}\right)\right] W\left(g_{x}^{h}\right) d g_{x}^{h}$, with $W\left(g_{x}^{h}\right)$ the $g$-factor Gaussian distribution. To describe the twopump experiment, discussed later, the second pulse perturbs the spin dynamics evolution at its arrival time $T_{D}$ in the same way as the first pulse, $\rho\left(T_{D}^{+}\right)=U_{\sigma^{+}}(\theta) \rho\left(T_{D}^{-}\right) U_{\sigma^{+}}^{+}(\theta)$.

Figure 1(b) shows the experimental and calculated PCD signals obtained at low temperature and $788 \mathrm{mT}$. The calculated PCD signal strongly depends on $T_{2}^{h}$ and on the Rabi angle $\theta$ when the other physical parameters involved in the calculation are fixed or known. To estimate experimentally $\theta$, the amplitude dependence of the $\operatorname{PCD}(t<0)$ signal on the excitation density has been studied. Figure 2(a) shows that, as for previous studies, ${ }^{23,24}$ the calculated curve has a maximum at $\theta \neq \pi$. The amplitude of the experimental signal reaches a maximum and then remains almost constant. This behavior is the signature of the $\theta$ inhomogeneity which avoids the observation of a maximum and a minimum at $\theta=2 \pi$, as predicted [the inset of Figure 2(a)]. A quite good agreement between the experimental and the calculated PCD curve is shown in Fig. 2(a) when a $\theta$ dispersion is considered, $\Delta \theta / \theta=40 \% .^{33}$ Results shown in Figures $1(\mathrm{~b})$ and $1(\mathrm{e})$ are obtained for $\theta \approx 0.9 \pi$. Figure $1(\mathrm{e})$ shows the sensitivity of the calculated $\operatorname{PCD}(t<0)$ curve to the $T_{2}^{h}$ value.

We have also split each pump pulse into two pulses with a fixed delay $T_{D}$ between them. Figure 3(a) shows PCD signals

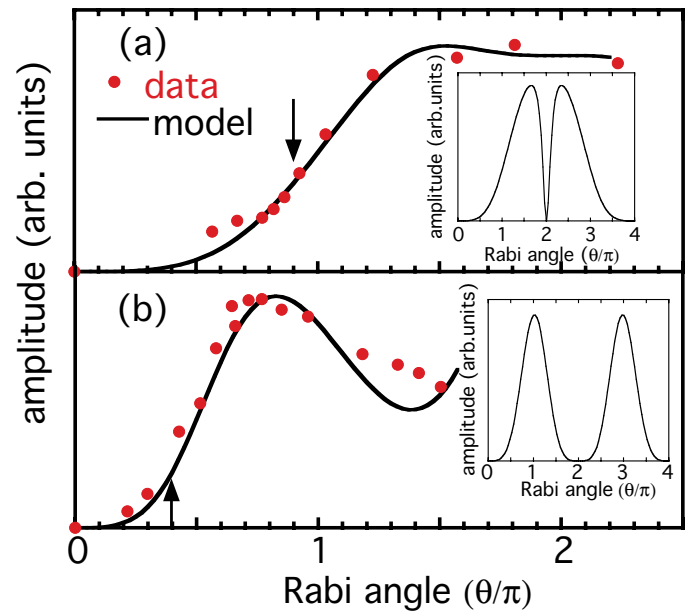

FIG. 2. (Color online) (a) Amplitude of $\operatorname{PCD}(t<0)$ for a singlepump excitation and (b) replica amplitude for a double-pump excitation vs the pump-pulse Rabi angle. Experiment: solid symbols; theoretical curve: solid line. The fitting curves are obtained with a $\theta$ dispersion of $40 \%$ and $55 \%$, respectively (corresponding to slightly different experimental conditions). Insets: Theoretical curves without $\theta$ dispersion. Arrows in (a) and (b) indicate the excitation conditions for data shown in Figs. 1(b) and 3(c), respectively.

for the case of a double-pump excitation with $T_{D}=T_{L} / 9$ and different field values. We observe that, as for experiments with a single-pump excitation, the coherent response of the synchronized QD ensemble is visible just before and after each pump pulse. Due to the weakness of the signal at negative delays, a magnification of the PCD is shown for clarity. Now, for a train of doublet pulses, the synchronization condition is more selective because it has to be extended also to the $T_{D}$ interval, $\omega_{h}=\mu_{B} g_{x}^{h} B_{x} / \hbar=2 \pi k / T_{D}$. We can distinguish two regimes in Fig. 3(a): the low- and moderate-magnetic-field regimes. The low-field regime [also represented in Fig. 3(b) for $B_{x}=60-100 \mathrm{mT}$ ] is characterized by the presence of an oscillatory signal after the second pump beam and at negative delays. As stated above, for a single-pump excitation, at low field, a relatively slow $T_{h}^{*}$ is responsible for a weak damping of the oscillations at negative delays. For moderate fields $(0.4 T \leqslant$ $\left.B_{x} \leqslant 1.2 T\right)$, as already evidenced for electrons, ${ }^{28}$ we observe a coherent hole-spin signal at a negative delay in the form of a replica at $-T_{D}$ of the first pump pulse. This replica is the result of the synchronization of a larger number of precession modes than in the curves shown in Fig. 3(b) and obtained in the low-field regime. Figure 3(c) shows this replica in more detail at $731 \mathrm{mT}$ for $T_{D}=T_{L} / 9$.

In low fields, in the range $60-120 \mathrm{mT}$, a strong selectivity of QD subsets participating in the PCD signal is obtained. A small modification of $B_{x}$ leads to a change in the oscillation amplitude, but does not affect their frequency [see Fig. 3(b)]: Only one subset of QDs meets the phase synchronization condition $\omega_{h}^{0}=2 \pi / T_{D}$ and makes a complete oscillation [fundamental synchronized mode (FSM)] just before the arrival of the second pump pulse. By modifying the $B_{x}$ value, we change the subset of QDs contributing to the synchronized signal. The higher is the magnetic field, the lower is the $\left|g_{x}^{h}\right|$ value of the FSM. In this way, we scan the broad distribution 

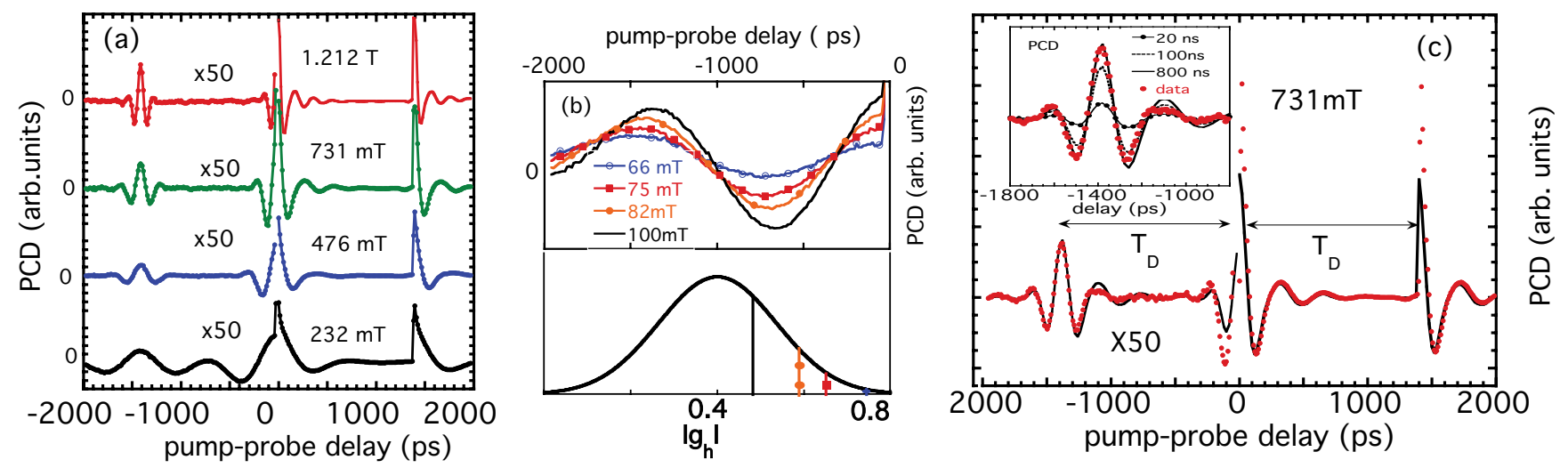

FIG. 3. (Color online) (a) PCD signal at $2 \mathrm{~K}$ vs pump-probe delay for different values of $B_{x}$ and for a double-pump excitation. The density of excitation for each pump was $\approx 1 \mathrm{~mW}$. The signal has been amplified $(50 \times)$ at negative delays. (b) Upper panel: $\operatorname{PCD}(t<0)$ vs pump-probe delay in the low-field regime. Lower panel: $\left|g_{x}^{h}\right|$ distribution; vertical lines indicate the $\left|g_{x}^{h}\right|$ value of FSM associated with $B_{x}$ shown in the upper panel. (c) PCD vs delay at $B_{x}=731 \mathrm{mT}$; experiment: solid symbols; theoretical curve: solid line; parameters are the same as in Fig. 1, with $\theta=0.4 \pi$ for both pumps. Inset: Magnification of the replica and comparison between experiments and theoretical curves for different $T_{2}^{h}$.

of $\left|g_{x}^{h}\right|$ factors in the high-value side, and at the same time the amplitude of the PCD signal follows the density of the FSM. We obtain, for example, that at $66 \mathrm{mT}$ only the subset of QDs with $\left|g_{x}^{h}\right|=0.74$ contributes to $\operatorname{PCD}(t<0)$. For $B_{x} \geqslant 120 \mathrm{mT}$, several QD subsets clearly contribute to the PCD signal, for example, two at $120 \mathrm{mT}$ and three at $220 \mathrm{mT}$ (with $\left|g_{x}^{h}\right|=0.22,0.44$, and 0.66).

As for a single-pump excitation, the calculated PCD signal depends strongly on $\theta$ and $T_{2}^{h}$. We have studied the dependence of the maximal oscillation amplitude of the replica on the pump density to determine the value of $\theta$ corresponding to the data of Fig. 3(c). The solid line in Fig. 2(b) is a fit to the experimental data with $\Delta \theta / \theta=55 \%$. The solid line in Fig. 3(c) shows the experimental and calculated PCD curve versus the pump-probe delay for $\theta=0.4 \pi$ and $T_{2}^{h}=0.8 \mu \mathrm{s}$; the agreement is very good. We estimate a lower bound to $T_{2}^{h}$ by determining the minimum value of $T_{2}^{h}$ that gives a good fit of the experimental PCD curves, in a temporal window of $4 \mathrm{~ns}$, for one [see Fig. 1(e)] and two pumps [see Fig. 3(c)] simultaneously. ${ }^{34}$ A constant value of $T_{2}^{h}=0.8 \mu \mathrm{s}$ was obtained in moderate $B_{x}$, which is in good agreement with recent spin-echo measurements ${ }^{18}$ and coherent population trapping. ${ }^{17}$ Moreover, this value is almost three times smaller than $T_{1}^{h}$ obtained in the same sample at similar magnetic fields. ${ }^{15}$ The hyperfine interaction has been identified at the origin of a dephasing time in the nanosecond range in the same sample. ${ }^{9}$ In the microsecond range, the coherence time is probably limited by extra nuclear-induced processes ${ }^{20}$ and/or impurities and an electrostatic environment of QDs. ${ }^{18,21}$ Further studies are required to determine the decoherence mechanisms for hole spins.

In conclusion, we have demonstrated the ability to reveal a long-living hole-spin coherence despite the fast dephasing imposed by a large dispersion of Larmor frequencies in a $p$-doped InAs QD ensemble. The determined $T_{2}^{h} \approx 0.8 \mu$ s confirms that the hole spin is a good candidate for a solid-state qubit. One-pump experiments synchronize the coherent evolution of several QD subsets to the periodic pulsed excitation, and twopump experiments go further in the selection of synchronized QD subsets. In particular, in the range 60-120 mT, a single subset of QDs is selected and the inhomogeneous distribution can be scanned by sweeping the applied magnetic field. Two-pump experiments also allow to control and manipulate the hole-spin coherence in an ensemble of QDs and then open perspectives for quantum information devices.

Note added. On completion of the publishing process, we became aware of a related publication, see Ref. 35 .

This work is supported by the QUAMOS Project No. ANR09-NANO-030 funded by the French National Agency (ANR).
*Present address: Department of Physics and Astronomy, University of Sheffield, Hicks Building, Hounsfield Road, Sheffield S3 7RH, United Kingdom.

${ }^{1}$ B. D. Gerardot, D. Brunner, P. A. Dalgarno, P. Öhberg, S. Seidl, M. Kroner, K. Karrai, N. G. Stoltz, P. M. Petroff, and R. J. Warburton, Nature (London) 451, 441 (2008).

${ }^{2}$ B. Eble, P. Desfonds, F. Fras, F. Bernardot, C. Testelin, M. Chamarro, A. Miard, and A. Lemaître, Phys. Rev. B 81, 045322 (2010)
${ }^{3}$ F. Fras, B. Eble, F. Bernardot, C. Testelin, M. Chamarro, A. Miard, and A. Lemaître, Appl. Phys. Lett. 100, 012104 (2012).

${ }^{4}$ P. Desfonds, B. Eble, F. Fras, C. Testelin, F. Bernardot, M. Chamarro, B. Urbaszek, T. Amand, X. Marie, J.-M. Gérard, V. Thierry-Mieg, A. Miard, and A. Lemaître, Appl. Phys. Lett. 96, 172108 (2010)

${ }^{5}$ A. J. Ramsay, S. J. Boyle, R. S. Kolodka, J. B. B. Oliveira, J. Skiba-Szymanska, H. Y. Liu, M. Hopkinson, A. M. Fox, and M. S. Skolnick, Phys. Rev. Lett. 100, 197401 (2008). 
${ }^{6}$ T. M. Godden, J. H. Quilter, A. J. Ramsay, Y. W. Wu, P. Brereton, I. J. Luxmoore, J. Puebla, A. M. Fox, and M. S. Skolnick, Phys. Rev. B 85, 155310 (2012).

${ }^{7}$ K. Müller, A. Bechtold, C. Ruppert, C. Hautmann, J. S. Wildmann, T. Kaldewey, M. Bichler, H. J. Krenner, G. Abstreiter, M. Betz, and J. J. Finley, Phys. Rev. B 85, 241306(R) (2012).

${ }^{8}$ J. Fischer, W. A. Coish, D. V. Bulaev, and D. Loss, Phys. Rev. B 78, 155329 (2008).

${ }^{9}$ B. Eble, C. Testelin, P. Desfonds, F. Bernardot, A. Balocchi, T. Amand, A. Miard, A. Lemaître, X. Marie, and M. Chamarro, Phys. Rev. Lett. 102, 146601 (2009).

${ }^{10}$ C. Testelin, F. Bernardot, B. Eble, and M. Chamarro, Phys. Rev. B 79, 195440 (2009).

${ }^{11}$ P. Fallahi, S. T. Yilmaz, and A. Imamoglu, Phys. Rev. Lett. 105, 257402 (2010).

${ }^{12}$ E. A. Chekhovich, A. B. Krysa, M. S. Skolnick, and A. I. Tartakovskii, Phys. Rev. Lett. 106, 027402 (2011).

${ }^{13}$ D. Heiss, S. Schaeck, H. Huebl, M. Bichler, G. Abstreiter, J. J. Finley, D. V. Bulaev, and D. Loss, Phys. Rev. B 76, 241306(R) (2007).

${ }^{14}$ F. Fras, B. Eble, P. Desfonds, F. Bernardot, C. Testelin, M. Chamarro, A. Miard, and A. Lemaître, Phys. Rev. B 84, 125431 (2011).

${ }^{15}$ F. Fras, B. Eble, P. Desfonds, F. Bernardot, C. Testelin, M. Chamarro, A. Miard, and A. Lemaître, Phys. Rev. B 86, 045306 (2012).

${ }^{16}$ Yan Li, N. Sinitsyn, D. L. Smith, D. Reuter, A. D. Wieck, D. R. Yakovlev, M. Bayer, and S. A. Crooker, Phys. Rev. Lett. 108, 186603 (2012).

${ }^{17}$ D. Brunner, B. D. Gerardot, P. A. Dalgarno, G. Wüst, K. Karrai, N. G. Stoltz, P. M. Petroff, and R. Warburton, Science 325, 70 (2009).

${ }^{18}$ K. De Greve, P. L. McMahon, D. Press, T. D. Ladd, D. Bisping, C. Schneider, M. Kamp, L. Worschech, S. Höfling, A. Forchel, and Y. Yamamoto, Nat. Phys. 7, 872, (2011).

${ }^{19}$ A. Greilich, S. G. Carter, D. Kim, A. S. Bracker, and D. Gammon, Nat. Photonics 5, 702 (2011).

${ }^{20}$ T. M. Godden, J. H. Quilter, A. J. Ramsay, Y. Wu, P. Brereton, S. J. Boyle, I. J. Luxmoore, J. Puebla-Nunez, A. M. Fox, and M. S. Skolnick, Phys. Rev. Lett. 108, 017402 (2012).
${ }^{21}$ D. Press, K. De Greve, P. L. McMahon, T. D. Ladd, B. Friess, C. Schneider, M. Kamp, S. Höfling, A. Forchel, and Y. Yamamoto, Nat. Photonics 4, 367 (2010).

${ }^{22}$ W. Yao, R. B. Liu, and L. J. Sham, Phys. Rev. B 74, 195301 (2006),

${ }^{23}$ A. Greilich, D. R. Yakovlev, A. Shabaev, Al. L. Efros, I. A. Yugova, R. Oulton, V. Stavarache, D. Reuter, A. Wieck, and M. Bayer, Science 313, 341 (2006); A. Schwan, S. Varwig, A. Greilich, D. R. Yakovlev, D. Reuter, A. D. Wieck, and M. Bayer, Appl. Phys. Lett. 100, 232107 (2012).

${ }^{24}$ I. A. Yugova, M. M. Glazov, D. R. Yakovlev, A. A. Sokolova, and M. Bayer, Phys. Rev. B 85, 125304 (2012).

${ }^{25}$ F. G. G. Hernandez, A. Greilich, F. Brito, M. Wiemann, D. R. Yakovlev, D. Reuter, A. D. Wieck, and M. Bayer, Phys. Rev. B 78, 041303(R) (2008).

${ }^{26}$ C. E. Pryor and M. E. Flatté, Phys. Rev. Lett. 96, 026804 (2006); 99, 179901(E) (2007).

${ }^{27}$ A. Schwan, B. M. Meiners, A. Greilich, D. R. Yakovlev, M. Bayer, A. D. B. Maia, A. A. Quivy, and A. B. Henriques, Appl. Phys. Lett. 99, 221914 (2011)

${ }^{28}$ A. Greilich, M. Wiemann, F. G. G. Hernandez, D. R. Yakovlev, I. A. Yugova, and M. Bayer, Phys. Rev. B 75, 23301 (2007).

${ }^{29}$ I. A. Merkulov, Al. L. Efros, and M. Rosen, Phys. Rev. B 65, 205309 (2002).

${ }^{30}$ E. Aubry, C. Testelin, F. Bernardot, M. Chamarro, and A. Lemaitre, Appl. Phys. Lett. 90, 242113 (2007)

${ }^{31}$ O. Krebs, P. Maletinsky, T. Amand, B. Urbaszek, A. Lemaître, P. Voisin, X. Marie, and A. Imamoglu, Phys. Rev. Lett. 104, 056603 (2010).

${ }^{32}$ S. A. Crooker, J. Brandt, C. Sandfort, A. Greilich, D. R. Yakovlev, D. Reuter, A. D. Wieck, and M. Bayer, Phys. Rev. Lett. 104, 036601 (2010).

${ }^{33}$ P. Borri, W. Langbein, S. Schneider, U. Woggon, R. L. Sellin, D. Ouyang, and D. Bimberg, Phys. Rev. B 66, 081306R (2002). The Rabi angle inhomogeneity in our case is not only determined by the inhomogeneity of the optical transition dipole moments but also by the profile of density of the laser spot on the sample.

${ }^{34}$ Because the laser repetition period is $13.1 \mathrm{~ns}$, theoretical curves are almost insensitive to $T_{2}^{h}$ values larger than $1 \mu \mathrm{s}$.

${ }^{35}$ S. Varwig, A. Schwan, D. Barmscheid, C. Müller, A. Greilich, I. A. Yugova, D. R. Yakovlev, D. Reuter, A. D. Wieck, and M. Bayer, Phys. Rev. B 86, 075321 (2012). 